Research Article

\title{
Global Existence of a Chemotactic Movement with Singular Sensitivity by Two Stimuli
}

\author{
Heping Ma $(\mathbb{D}$ \\ School of Science, Hubei University of Technology, Wuhan 430068, Hubei, China \\ Correspondence should be addressed to Heping Ma; 1049187340@qq.com
}

Received 29 April 2021; Revised 11 July 2021; Accepted 28 July 2021; Published 5 August 2021

Academic Editor: Shiping Wen

Copyright (C) 2021 Heping Ma. This is an open access article distributed under the Creative Commons Attribution License, which permits unrestricted use, distribution, and reproduction in any medium, provided the original work is properly cited.

In this study, we deal with the chemotaxis system with singular sensitivity by two stimuli under homogeneous Neumann boundary conditions in a bounded domain with smooth boundary. Under appropriate regularity assumptions on the initial data, we show that the system possesses global classical solution. Our results generalize and improve previously known ones.

\section{Introduction}

Chemotaxis is a well-known biological phenomenon describing the collective motion of cells or the evolution of density of bacteria driven by chemicals, including embryo development, skin wound healing, cancer invasion, and metastasis. The pioneering works of the chemotaxis model was introduced by Keller and Segel in [1], describing the aggregation of cellular slime mold toward a higher concentration of a chemical signal, which reads

$$
\begin{cases}u_{t}=\Delta u-\nabla \cdot(u \nabla v), & x \in \Omega, t>0, \\ v_{t}=\Delta v-v+u, & x \in \Omega,\end{cases}
$$

where $\Omega \subset \mathbb{R}^{N}(N \geq 1)$ is a bounded domain with smooth boundary. The mathematical analysis of (1) and the variants thereof mainly concentrate on the boundedness and blowup of the solutions (refer to [2-6] and the references therein), that is, it is well-known that for all suitably regular initial data $\left(u_{0}, v_{0}\right)$, an associated Neumann initial boundary value problem, posed in a smooth $N$-dimensional domain $\Omega$, Osaki and Yagi [4] proved that system (1) always possesses a global bounded classical solution in one-dimensional bounded domain, Nagai et al. [3] showed that system (1) also admits a global bounded classical solution in twodimensional bounded domain if $\left\|u_{0}\right\|_{L^{1}(\Omega)}$ is small, as $N \geq 3$, Winkler [5] proved that for each $q>(N / 2), p>N$, one can find $\varepsilon_{0}>0$, such that if the initial data $\left(u_{0}, v_{0}\right)$ fulfill
$\left\|u_{0}\right\|_{L^{q}(\Omega)}<\varepsilon$ and $\left\|v_{0}\right\|_{L^{p}(\Omega)}<\varepsilon$ for some $\varepsilon<\varepsilon_{0}$, then the solution is global in time and bounded; this is complemented by corresponding findings on the occurrence of finite-time blow-up of some solutions emanating from smooth but appropriately large initial data $[2,6]$. In the past few decades, system (1) has attracted extensive attentions.

Keller and Segel [7] introduced a phenomenological model of the wave-like solution behavior without any type of cell kinetics, a prototypical version of which is given by

$$
\begin{cases}u_{t}=\Delta u-\chi \nabla \cdot\left(\frac{u}{v} \nabla v\right), & x \in \Omega, t>0, \\ v_{t}=\Delta v-u v, & x \in \Omega, t>0 .\end{cases}
$$

where $u$ represents the density of bacteria and $v$ denotes the concentration of the nutrient. The second equation models consumption of the signal. In the first equation, the chemotactic sensitivity is determined according to the Weber-Fechner law, which says that the chemotactic sensitivity is proportional to the reciprocal of signal density. Winkler [8] proved that if initial data satisfy appropriate regularity assumptions, system (2) possesses at least one global generalized solution in two-dimensional bounded domains. Moreover, he took into account asymptotic behavior of solutions to system (2) and proved that $v(\cdot, t) \vec{\longrightarrow} 0$ in $L^{\infty}(\Omega)$ and $v(\cdot, t) \longrightarrow 0$ in $L^{p}(\Omega)$ as $t \longrightarrow \infty$ provided $\int_{\Omega} u_{0} \leq m,-\int_{\Omega} \ln \left(v_{0} /\left\|v_{0}\right\|_{L^{\infty}(\Omega)}\right) \leq M$, where $m, M$ are the 
positive constants. When $u v$ is replaced by $g(u) v$, $g \in C^{1}(\mathbb{R})$, and $0 \leq g(u) \leq u^{\beta}, \beta \in(0,1), \chi \in(0,1)$, and any sufficiently regular initial data, Lankeit and Viglialoro [9] showed that system (2) has a global classical solution. Moreover, if additionally $m=\left\|u_{0}\right\|_{L^{1}(\Omega)}$ is sufficiently small, then also their boundedness is achieved. When system (2) has a logistic source $f(u)$, Lankeit and Lankeit [10] showed that system (2) possesses a global generalized solution for any $\chi \geq 0, \quad r \geq 0$, and $\mu>0$ if $f(u)=\kappa u-\mu u^{2}$. As $f(u)=r u-\mu u^{k}$, and $1 / v$ is replaced by $\phi(v)$, $\phi(v) \in C^{1}(0, \infty)$, satisfying $\phi(v) \longrightarrow 0$ as $v \longrightarrow \infty$. Zhao and Zheng [11] proved that system (2) possesses a unique positive global classical solution provided $k>1$ with $N=1$ or $k>1+(N / 2)$ with $N \geq 2$. When $1 / v$ is replaced by $1 / v^{\alpha}$, $\alpha \geq 1$ and $\Delta v$ turned into $\varepsilon \Delta v$, Zhao et al. [12] obtained the global existence of classical solutions with $N=1$. Moreover, for any global classical solution $(u, v)$ to the case of $N$, it is shown that $v$ converges to 0 in the $L^{\infty}$-norm as $t \longrightarrow \infty$ with decay rate established whenever $\varepsilon \in\left(\varepsilon_{0}, 1\right)$ with $\varepsilon_{0}=\max \left\{0,1-\left(\chi /\left(\alpha\left\|v_{0}\right\|_{L^{\infty}(\Omega)}^{\alpha-1}\right)\right)\right\}$. When $u$ is replaced by $f(u)$ and $0<f(u) \leq K(u+1)^{\alpha}$, Liu [13] showed that for any sufficiently smooth initial data, system (2) admits a global classical solution when either $N=1$ and $\alpha<2$ or $N \geq 2$ and $\alpha<1-(N / 4)$. When $\Delta u$ is replaced by $\Delta u^{m}(m \geq 1)$, Lankeit [14] proved that if $m>1+(N / 4)$, system (2) admits a global classical solution or global locally bounded weak solution.

This study deals with a chemotaxis system with singular sensitivity by two stimuli, which is given by

$$
\begin{cases}u_{t}=\nabla \cdot\left((u+1)^{m-1} \nabla u\right)-\nabla \cdot\left(\frac{\phi_{1}(u)}{v} \nabla v\right)+\nabla \cdot\left(\frac{\phi_{2}(u)}{w} \nabla w\right), & x \in \Omega, t>0, \\ v_{t}=\Delta v-u v, & x \in \Omega, t>0, \\ w_{t}=\Delta w-u w, & x \in \Omega, t>0, \\ \frac{\partial u}{\partial \nu}=\frac{\partial v}{\partial \nu}=\frac{\partial w}{\partial \nu}=0, & x \in \partial \Omega, t>0, \\ u(x, 0)=u_{0}(x), v(x, 0)=v_{0}(x), w(x, 0)=w_{0}(x), & x \in \Omega,\end{cases}
$$

where $\Omega \subset \mathbb{R}^{N}(N \geq 2)$ is a bounded domain with smooth boundary $\partial \Omega,(\partial / \partial \nu)$ denotes the derivative with respect to the outer normal of $\partial \Omega$, and $u(x, t), v(x, t)$, and $w(x, t)$ represent the density of the cell population, the concentration of the chemoattractant substances, and the concentration of the chemorepellent substances, respectively. We assume that $m \geq 1, \phi_{1}(u), \phi_{2}(u) \in C^{1+\sigma}([0, \infty))$, where $\sigma \in(0,1)$ and satisfies

$$
\begin{aligned}
& 0<\phi_{1}(s) \leq \Gamma_{1} s^{\alpha_{1}}, \\
& \phi_{1}(0)=0, \\
& 0<\phi_{2}(s) \leq \Gamma_{2} s^{\alpha_{2}}, \\
& \phi_{2}(0)=0, \quad \text { for all } s>0,
\end{aligned}
$$

with $\Gamma_{1}, \Gamma_{2}>0$, and $\alpha_{1}, \alpha_{2}<2\left(\alpha_{1} \neq \alpha_{2}\right)$ are the constants. Furthermore, we assume that the initial data $\left(u_{0}, v_{0}, w_{0}\right)$ satisfy

$$
\begin{cases}u_{0} \in C^{0}(\bar{\Omega}), & u_{0} \geq 0 \text { in } \Omega \text { and } u_{0} \equiv 0 \text { as well as } \\ v_{0}, w_{0} \in W^{1, \infty}(\Omega), & v_{0}(x), w_{0}(x)>0 \text { in } \bar{\Omega}\end{cases}
$$

It is different from model (3); the following is the attraction-repulsion Keller-Segel model where the signal is produced and not consumed by the cells:

$$
\begin{cases}u_{t}=\nabla \cdot(D(u) u)-\chi \nabla \cdot(u \nabla v)+\xi \nabla \cdot(u \nabla w), & x \in \Omega, t>0, \\ \tau_{1} v_{t}=\Delta v+\alpha u-\beta v, & x \in \Omega, t>0, \\ \tau_{2} w_{t}=\Delta w+\gamma u-\delta w, & x \in \Omega, t>0,\end{cases}
$$

which was proposed in [15] to describe how the combination of chemical might interact to produce aggregates of cells. In two space dimensions, when $D(u)=1, \tau_{1}=1$, and $\tau_{2}=0$, Jin et al. [16] proved that if $\chi \alpha-\xi \gamma \leq 0$, then system (6) possesses a unique global uniformly in-time bounded classical solution, and if $\chi \alpha-\xi \gamma>0, \int_{\Omega} u_{0}(x)<(4 \pi /$ $(\chi \alpha-\xi \gamma))$, the same result is also obtained; to the contrary, if $\int_{\Omega} u_{0}(x)>(4 \pi /(\chi \alpha-\xi \gamma))$, the solutions blow up in finite or infinite time. Ulteriorly, $\mathrm{Xu}$ et al. [17] showed that if $\xi \gamma \beta \geq \chi \alpha \delta$, the global classical solution of system (6) converged to the unique constant state $\left(\bar{u}_{0},(\alpha / \beta) \bar{u}_{0},(\gamma / \delta) \bar{u}_{0}\right)$ as $t \longrightarrow \infty$, where $\bar{u}_{0}=(1 /|\Omega|) \int_{\Omega} u_{0}$. When $D(u)=1$, $\tau_{1}=\tau_{2}=0$, Li et al. [18] proved that if $\int_{\Omega} u_{0}(x)\left|x-x_{0}\right|^{2}$ is sufficiently small, either of the following cases holds: (i) $\chi \alpha>\xi \gamma, \delta \geq \beta$, and $\int_{\Omega} u_{0}(x)>(8 \pi /(\chi \alpha-\xi \gamma))$; (ii) $\chi \alpha \delta>\xi \gamma \beta$, $\delta<\beta$, and $\int_{\Omega} u_{0}(x)>(8 \pi /(\chi \alpha \delta-\xi \gamma \beta))$, the corresponding solution of (6) blows up in finite time. Hu et al. [19] showed that if $\chi \alpha>\xi \gamma$ and $\int_{\Omega} u_{0}(x)>(8 \pi /(\chi \alpha-\xi \gamma))$ hold with $\int_{\Omega} u_{0}(x)\left|x-x_{0}\right|^{2}$ small enough, the solution of (6) blows up in finite time. In high dimensions, when $D(u)=1$, $\tau_{1}=\tau_{2}=1$, Jin [20] proved that if $\xi \gamma>\chi \alpha$, system (6) possesses global classical solution in dimensions and weak 
solution in three dimensions with large initial data. When $\tau_{1}=1, \tau_{2}=0, D(s) \geq 0, s \geq 0$, and $D(s)=D_{0}(s) s^{-\theta}$ for all $s>0$ with some $D_{0}>0$ and $\theta \in \mathbb{R}$, Lin et al. [21] showed that if $\theta<(2 / N)-1$, for any nonnegative initial data, system (6) admits a unique classical solution which was global and bounded; if $\theta>(2 / N)-1$, the radially symmetric solutions may blow up in finite time. When $\tau_{1}, \tau_{2}>0, D(u)>0$ for $u \geq 0$ and $D(u) \geq \mathrm{d} u^{m-1}$ with $d>0$ and $m \geq 1$ for all $u>1, \mathrm{Li}$ et al. [22] proved that the corresponding initial boundary value problem possesses a unique global bounded classical solution for $m>2-(2 / N)$. In particular, in the case $\tau_{1}=\tau_{2}$ and $\chi \alpha=\xi \gamma$, the solution is globally bounded if $m>2-(2 / N)-\left((N+2) /\left(N^{2}-N+2\right)\right)$. When the system has a logistic source, the relevant results can be found in [23-25].

To the best of our knowledge, Dong et al. [26] first put forward the following chemotactic model with general rotational sensitivity caused by two stimuli:

$$
\begin{cases}u_{t}=\Delta u-\nabla \cdot\left(u S_{1}(x, u, v, w) \nabla v\right)+\nabla \cdot\left(u S_{2}(x, u, v, w) \nabla w\right), & x \in \Omega, t>0, \\ v_{t}=\Delta v-u v, & x \in \Omega, t>0 \\ w_{t}=\Delta w-u w, & x \in \Omega, t>0\end{cases}
$$

where $\Omega \subset \mathbb{R}^{N}(N \geq 1)$ is a bounded domain with smooth boundary $\partial \Omega$. Under mild assumptions on $S_{1}(x, u, v, w)$, $S_{2}(x, u, v, w)$, system (7) admits at least one global generalized solution.

Throughout above analysis, compared with system (6), the theory of system (3) is so fragmentary. To the best of our knowledge, the global classical solution of model (3) in $N \geq 2$ has never been touched. No matter biological relevance or mathematical meaning, we find it is worth addressing the basic solvability theory of the model (3). Inspired by the arguments in previous studies $[8,13,14,26,27]$, we mainly investigate the global classical solution in a chemotactic movement with singular sensitivity by two stimuli. Theorem 1 partially generalizes and improves previously known ones.

In this study, we use symbols $C_{i}$ and $c_{i}(i=1,2, \ldots)$ as some generic positive constants which may vary in the context. For simplicity, $u(x, t)$ is written as $u$, the integral $\int_{\Omega} u(x) \mathrm{d} x$ is written as $\int_{\Omega} u(x)$, and $\int_{0}^{t} \int_{\Omega} u(x) \mathrm{d} x \mathrm{~d} t$ is written as $\int_{0}^{t} \int_{\Omega} u(x)$.
The rest of this study is organized as follows. In Section 2, we summarize some useful lemmata in order to prove the main result. In Section 3, we give some fundamental estimates for the solution to system (3) and proof of Theorem 1.

\section{Preliminaries and Main Result}

In this section, we give the main theorem and the local existence of the classical solution to (3) and also summarize some useful lemmata in order to prove the main result. Noting the singular chemotaxis term, we let

$$
\begin{aligned}
& n_{1}:=-\ln \left(\frac{v(x, t)}{\left\|v_{0}\right\|_{L^{\infty}(\Omega)}}\right), \\
& n_{2}:=-\ln \left(\frac{w(x, t)}{\left\|w_{0}\right\|_{L^{\infty}(\Omega)}}\right), \quad(x, t) \in \bar{\Omega} \times[0, \infty) .
\end{aligned}
$$

Then, we can rewrite (3) as

$$
\begin{cases}u_{t}=\nabla \cdot\left((u+1)^{m-1} \nabla u\right)+\nabla \cdot\left(\phi_{1}(u) \nabla n_{1}\right)-\nabla \cdot\left(\phi_{2}(u) \nabla n_{2}\right), & x \in \Omega, t>0, \\ n_{1 t}=\Delta n_{1}-\left|\nabla n_{1}\right|^{2}+u, & x \in \Omega, t>0, \\ n_{2 t}=\Delta n_{2}-\left|\nabla n_{2}\right|^{2}+u, & x \in \Omega, t>0, \\ \frac{\partial u}{\partial \nu}=\frac{\partial n_{1}}{\partial \nu}=\frac{\partial n_{2}}{\partial \nu}=0, & x \in \partial \Omega, t>0, \\ u(x, 0)=u_{0}(x), n_{1}(x, 0)=n_{10}(x):=-\ln \left(\frac{v(x, t)}{\left\|v_{0}\right\|_{L^{\infty}(\Omega)}}\right), & \\ n_{2}(x, 0)=n_{20}(x):=-\ln \left(\frac{w(x, t)}{\left\|w_{0}\right\|_{L^{\infty}(\Omega)}}\right), & x \in \Omega .\end{cases}
$$


At first, we give the main result of global existence of the classical solution to (3).

Theorem 1. Let $\Omega \subset \mathbb{R}^{N}(N \geq 2)$ be a bounded domain with smooth boundary. Assume that $\min \{1-(2 / N), 2-(6 /$ $(N+2))\}<m \leq 2-(2 / N), \phi_{1}(u), \phi_{2}(u)$ satisfy (4) and

$$
\begin{aligned}
& \alpha_{1}<1-\frac{(1-m) N}{4}, \\
& \alpha_{2}<1-\frac{(1-m) N}{4} .
\end{aligned}
$$

Then, for any choice of the initial data $\left(u_{0}, v_{0}, w_{0}\right)$ fulfilling (5), there exists a triple $(u, v, w) \in\left(C^{0}(\bar{\Omega} \times t\right.$ $[0, \infty)) \cap C^{2,1}(\bar{\Omega} \times(0, \infty))^{3}$ which solves (3) classically. Moreover, we have $u \geq 0$ and $v, w>0$ in $\bar{\Omega} \times[0, \infty)$.

Remark 1. Theorem 1 shows that system (3) admits a global classical solution nothing to do with $\Gamma_{1}$ and $\Gamma_{2}$.

Remark 2. Theorem 1 partially generalizes and improves the results in ([14], Theorem 1.1) and ([13], Theorem 1.1).

Remark 3. If we replaced the terms $u v$ and $u w$ in system (3) by $u f_{1}(v, w)$ and $u f_{2}(v, w)$, respectively, Theorem 1 still holds provided that both $f_{1}$ and $f_{2}$ are nonnegative differentiable functions satisfying $f_{1}(0,0)=0$ and $f_{2}(0,0)=0$.

In the sequel, we will consider system (9) to obtain the local boundedness of $v, w$ and then come back to system (3) to prove the main theorem. Under the framework of fixed point theorem, we will prove the local existence of classical solution to system (3) in the following lemma. The proof is quite standard, and a more detailed display of a similar reasoning in a related circumstance can be found in [14].

Lemma 1. Let $\Omega \subset \mathbb{R}^{N}(N \geq 2)$ be a bounded domain with smooth boundary. Assume that $m \geq 1, \phi_{1}(u), \phi_{2}(u)$ satisfy (4) and $\alpha_{1}<2, \alpha_{2}<2$. Then, for any initial data $\left(u_{0}, v_{0}, w_{0}\right)$ fulfilling (5), there exist $T_{\max } \in(0, \infty]$ and a triple $(u, v, w) \in\left(C^{0}\left(\bar{\Omega} \times\left[0, T_{\max }\right)\right) \cap C^{2,1}\left(\bar{\Omega} \times\left(0, T_{\max }\right)\right)^{3}\right.$ solving (3) classically in $\Omega \times\left(0, T_{\max }\right)$, where $T_{\max }$ denotes the maximal existence time. Moreover, the solution $(u, v, w)$ satisfies

$$
\begin{aligned}
\int_{\Omega} u(x, t) & =\int_{\Omega} u_{0}(x), \quad \text { for all } t>0, \\
0 & <v<\left\|v_{0}\right\|_{L^{\infty}(\Omega)}, \\
0 & <w<\left\|w_{0}\right\|_{L^{\infty}(\Omega)} \text { in } \Omega \times(0, \infty),
\end{aligned}
$$

if $T_{\max }<+\infty$, then

$$
\limsup _{t>T_{\max }}\|u(\cdot, t)\|_{L^{\infty}(\Omega)}=\infty .
$$

Lemma 2. The solution of (9) satisfies

$$
\begin{aligned}
& \int_{0}^{t} \int_{\Omega}\left|\nabla n_{1}\right|^{2} \leq \int_{\Omega} n_{10}+C t, \quad \text { for all } t \in\left(0, T_{\max }\right), \\
& \int_{0}^{t} \int_{\Omega}\left|\nabla n_{2}\right|^{2} \leq \int_{\Omega} n_{20}+C t, \quad \text { for all } t \in\left(0, T_{\max }\right),
\end{aligned}
$$

where $C=\int_{\Omega} u_{0}(x)$.

Proof. Integrating the second equation of (9) with respect to $(x, t) \in \Omega \times\left(0, T_{\max }\right)$, we have

$$
\begin{aligned}
\int_{\Omega} n_{1}-\int_{\Omega} n_{10} & =-\int_{0}^{t} \int_{\Omega}\left|\nabla n_{1}\right|^{2}+\int_{0}^{t} \int_{\Omega} u \\
& \leq-\int_{0}^{t} \int_{\Omega}\left|\nabla n_{1}\right|^{2}+C t
\end{aligned}
$$

Due to the positivity of $n_{1}$, we obtain (13) immediately. Likewise, we get (14). The proof is complete.

Lemma 3 (Gagliardo-Nirenberg interpolation inequality [28]). Let $0<\theta \leq p \leq(2 N /(N-2))$. There exists a positive constant $C_{G N}$, such that for all $u \in W^{1,2}(\Omega) \cap L^{\theta}(\Omega)$,

$$
\|u\|_{L^{p}(\Omega)} \leq C_{G N}\left(\|\nabla u\|_{L^{2}(\Omega)}^{a}\|u\|_{L^{\theta}(\Omega)}^{1-a}+\|u\|_{L^{\theta}(\Omega)}\right),
$$

is valid with $a=(((N / \theta)-(N / p)) /(1-(N / 2)+$ $(N / \theta))) \in(0,1)$.

Lemma 4 (See [29]). Let $T>0$ and $y \in C^{0}([0, T)) \cap C^{1}(0$, T) be such that

$$
y^{\prime}(t)+a y(t) \leq g(t), \quad \text { for all } t \in(0, T),
$$

where $g \in L_{\text {loc }}^{1}(\mathbb{R})$ has the property that

$$
\frac{1}{\tau} \int_{t}^{t+\tau} g(s) \mathrm{d} s \leq b, \quad \text { for all } t \in(0, T),
$$

with some $\tau>0$ and $b>0$. Then,

$$
y(t) \leq y(0)+\frac{b \tau}{1-e^{-a \tau}}, \quad \text { for all } t \in[0, T) .
$$

\section{Proof of Theorem 1}

In this section, we establish some priori estimates for solutions to system (9); we first establish a bound for $n_{1}(x, t)$ and $n_{2}(x, t)$ in the one-dimensional case, which differs from that in the multidimensional settings.

Lemma 5. Let $N \geq 2$ and suppose that $\min \{1-(2 / N)$, $2-(6 /(N+2))\}<m \leq 2-(2 / N)$, (4) holds with $\sigma \in(0,1)$, $\Gamma_{1}, \Gamma_{2}>0$, and $\alpha_{1}<1-((1-m) N / 4), \quad \alpha_{2}<1-((1-m)$ $N / 4)$. Then, there exists $C_{3}, C_{4}>0$, such that the solution of (9) satisfies 


$$
\begin{gathered}
\int_{\Omega} u^{1-2 \alpha_{1}} \leq C_{3}, \\
\int_{\Omega} u^{1-2 \alpha_{2}} \leq C_{4}, \quad \text { for all } t \in\left(0, T_{\max }\right), \\
\int_{0}^{t} \int_{\Omega} u^{m-2 \alpha_{1}-1}|\nabla u|^{2} \leq C_{3}(1+t), \\
\int_{0}^{t} \int_{\Omega} u^{m-2 \alpha_{2}-1}|\nabla u|^{2} \leq C_{4}(1+t), \quad \text { for all } t \in\left(0, T_{\max }\right) .
\end{gathered}
$$

Proof. Multiplying the first equation in (9) by $2\left(1-\alpha_{1}\right) u^{1-2 \alpha_{1}}$, integrating by parts, using (4) and Young's inequality, we have

$$
\begin{aligned}
\frac{\mathrm{d}}{\mathrm{d} t} \int_{\Omega} u^{2-2 \alpha_{1}}= & 2\left(1-\alpha_{1}\right) \int_{\Omega} u^{1-2 \alpha_{1}}\left(\nabla \cdot\left((u+1)^{m-1} \nabla u\right)+\nabla \cdot\left(\phi_{1}(u) \nabla n_{1}\right)-\nabla \cdot\left(\phi_{2}(u) \nabla n_{2}\right)\right) \\
\leq & -L_{1} \int_{\Omega} u^{m-2 \alpha_{1}-1}|\nabla u|^{2}-2 L_{1} \int_{\Omega} u^{-2 \alpha_{1}} \phi_{1}(u) \nabla u \cdot \nabla n_{1} \\
& +2 L_{1} \int_{\Omega} u^{-2 \alpha_{1}} \phi_{2}(u) \nabla u \cdot \nabla n_{2} \\
\leq & -2 L_{1} \int_{\Omega} u^{m-2 \alpha_{1}-1}|\nabla u|^{2}+\frac{1}{2} L_{1} \int_{\Omega} u^{m-2 \alpha_{1}-1}|\nabla u|^{2} \\
& +2 L_{1} \int_{\Omega} u^{1-2 \alpha_{1}-m} \phi_{1}^{2}(u)\left|\nabla n_{1}\right|^{2}+\frac{1}{2} L_{1} \int_{\Omega} u^{m-2 \alpha_{1}-1|\nabla u|^{2}} \\
& +2 L_{1} \int_{\Omega} u^{1-2 \alpha_{1}-m} \phi_{2}^{2}(u)\left|\nabla n_{2}\right|^{2} \\
\leq & -L_{1} \int_{\Omega} u^{1-2 \alpha_{1}-m}|\nabla u|^{2}+2 L_{1} \Gamma_{1} \int_{\Omega} u^{1-m}\left|\nabla n_{1}\right|^{2} \\
& +2 L_{1} \Gamma_{2} \int_{\Omega} u^{1+2 \alpha_{2}-2 \alpha_{1}-m}\left|\nabla n_{2}\right|^{2}
\end{aligned}
$$

for all $t \in\left(0, T_{\max }\right)$, where $L_{1}=\left(1-\alpha_{1}\right)\left(1-2 \alpha_{1}\right)$, and we have used the facts that $L_{1}>0$ due to $\alpha_{1}<1-((1-m) N / 4)$; since $\alpha_{1} \neq \alpha_{2}$, without loss of generality, we let $\alpha_{1}>\alpha_{2}$; by

Lemma 1 , we know that there exists a constant $\delta_{1}>0$, such that $u>\delta_{1}$, and with some rearrangements, we have

$$
\frac{\mathrm{d}}{\mathrm{d} t} \int_{\Omega} u^{2-2 \alpha_{1}}+L_{1} \int_{\Omega} u^{-2 \alpha_{1}}|\nabla u|^{2} \leq 2 \delta_{1}^{1+2 \alpha_{2}-2 \alpha_{1}-m} L_{1} \Gamma_{2} \int_{\Omega}\left|\nabla n_{2}\right|^{2}+2 \delta_{1}^{1-m} L_{1} \Gamma_{1} \int_{\Omega}\left|\nabla n_{1}\right|^{2} .
$$

Multiplying the first equation in (9) by $2\left(1-\alpha_{2}\right) u^{1-2 \alpha_{2}}$, similar to (23), we have

$$
\begin{aligned}
\frac{\mathrm{d}}{\mathrm{d} t} \int_{\Omega} u^{2-2 \alpha_{2}}+L_{2} \int_{\Omega} u^{m-2 \alpha_{2}-1}|\nabla u|^{2} \leq & 2 L_{2} \Gamma_{1} \int_{\Omega} u^{1+2 \alpha_{1}-2 \alpha_{2}-m}\left|\nabla n_{1}\right|^{2} \\
& +2 \delta_{1}^{1-m} L_{2} \Gamma_{2} \int_{\Omega}\left|\nabla n_{2}\right|^{2}
\end{aligned}
$$


where by Lemma 2 , we know that there exists a constant $c_{1}>0$, such that $\|u\|_{L^{1}(\Omega)} \leq c_{1}$.

$$
u^{1+2 \alpha_{1}-2 \alpha_{2}-m} \leq \begin{cases}c_{1}^{1+2 \alpha_{1}-2 \alpha_{2}-m}, & \text { if } m \leq 1+2 \alpha_{1}-2 \alpha_{2}, \\ \delta_{1}^{1+2 \alpha_{1}-2 \alpha_{2}-m}, & \text { if } m>1+2 \alpha_{1}-2 \alpha_{2} .\end{cases}
$$

$$
\frac{\mathrm{d}}{\mathrm{d} t} \int_{\Omega} u^{2-2 \alpha_{2}}+L_{2} \int_{\Omega} u^{m-2 \alpha_{2}-1}|\nabla u|^{2} \leq 2 c_{2} L_{2} \Gamma_{1} \int_{\Omega}\left|\nabla n_{1}\right|^{2}+2 \delta_{1}^{1-m} L_{2} \Gamma_{2} \int_{\Omega}\left|\nabla n_{2}\right|^{2} .
$$

Combining with (23) and (26), we obtain that

$$
\begin{aligned}
& \frac{\mathrm{d}}{\mathrm{d} t} \int_{\Omega} u^{2-2 \alpha_{1}}+\frac{\mathrm{d}}{\mathrm{d} t} \int_{\Omega} u^{2-2 \alpha_{2}}+L_{1} \int_{\Omega} u^{m-2 \alpha_{2}-1}|\nabla u|^{2}+L_{2} \int_{\Omega} u^{m-2 \alpha_{2}-1}|\nabla u|^{2} \\
& \leq 2 \Gamma_{1}\left(\delta_{1}^{1-m} L_{1}+c_{2} L_{2}\right) \int_{\Omega}\left|\nabla n_{1}\right|^{2}+2 \Gamma_{2}\left(\delta_{1}^{1-m} L_{2}+\delta_{1}^{1+2 \alpha_{2}-2 \alpha_{1}-m} L_{1}\right) \int_{\Omega}|\nabla n|_{2}^{2} .
\end{aligned}
$$

By the Gagliardo-Nirenberg inequality with (16), there exist $c_{3}, c_{4}>0$, such that

$$
\begin{aligned}
& \int_{\Omega} u^{2-2 \alpha_{1}}=\left\|u^{\left(m-2 \alpha_{2}+1\right) / 2}\right\|_{L^{\left.2\left(2-2 \alpha_{1}\right) /\left(m-2 \alpha_{2}+1\right)\right)(\Omega)}}^{\left(2\left(2-2 \alpha_{1}\right) /\left(m-2 \alpha_{2}+1\right)\right)} \\
& \leq c_{3}\left\|\nabla u^{\left(\left(m-2 \alpha_{2}+1\right) / 2\right)}\right\|_{L^{2}(\Omega)}^{\left(2\left(2-2 \alpha_{1}\right) /\left(m-2 \alpha_{2}+1\right)\right) \theta_{1}}\left\|u^{\left(\left(m-2 \alpha_{2}+1\right) / 2\right)}\right\|_{L^{\left(2 /\left(m-2 \alpha_{2}+1\right)\right)(\Omega)}}^{\left(2\left(2-2 \alpha_{1}\right) /\left(m-2 \alpha_{2}+1\right)\right)\left(1-\theta_{1}\right)} \\
&+c_{3}\left\|u^{\left(\left(m-2 \alpha_{2}+1\right) / 2\right)}\right\|_{L^{\left(2 /\left(m-2 \alpha_{2}+1\right)\right)}(\Omega)}^{\left(2\left(2-2 \alpha_{1}\right) /\left(m-2 \alpha_{2}+1\right)\right)} \\
& \leq c_{4} \int_{\Omega} u^{m-2 \alpha_{1}-1}|\nabla u|^{2}+c_{4}, \quad \\
& y^{\prime}(t)+y(t)+c_{6} \int_{\Omega} u^{-2 \alpha_{1}}|\nabla u|^{2}+c_{6} \int_{\Omega} u^{-2 \alpha_{2}}|\nabla u|^{2} \leq c_{7} g(t) .
\end{aligned}
$$

with

$$
\theta_{1}=\frac{\left(\left(m-2 \alpha_{2}-1\right) N / 2\right)-\left(\left(m-2 \alpha_{2}-1\right) N / 4\left(1-\alpha_{1}\right)\right)}{1-(N / 2)+\left(\left(m-2 \alpha_{2}-1\right) N / 2\right)} \in(0,1),
$$

due to $\alpha_{1}<1-((1-m) N / 4)$. Similar to (28), there exists $c_{5}>0$, such that

$$
\int_{\Omega} u^{2-2 \alpha_{2}} \leq c_{5} \int_{\Omega} u^{m-2 \alpha_{2}-1}|\nabla u|^{2}+c_{5} .
$$

Choose appropriate $c_{4}, c_{5}$, such that $c_{4} \leq L_{1}$ and $c_{5} \leq L_{2}$. Let $y(t)=\int_{\Omega} u^{2-2 \alpha_{1}}+\int_{\Omega} u^{2-2 \alpha_{2}}, \quad g(t)=\int_{\Omega}\left|\nabla n_{1}\right|^{2}+\int_{\Omega} \mid \nabla$ $\left.n_{2}\right|^{2}$,

$c_{6}=\min \left\{L_{1}-c_{4}, L_{2}-c_{5}\right\}$,

$c_{7}=\max \left\{2 \Gamma_{1}\left(\delta_{1}^{1-m} L_{1}+c_{2} L_{2}\right), 2 \Gamma_{2}\left(\delta_{1}^{1-m} L_{2}+\delta_{1}^{1+2 \alpha_{2}-2 \alpha_{1}-m} L_{1}\right)\right\}$.

As from Lemma 3 and Lemma 4, we readily obtain (28). (21) follows by integrating (32) in time. The proof is complete.

Lemma 6. Let $N \geq 2$ and suppose that $\min \{1-(2 / N)$, $2-(6 /(N+2))\}<m \leq 2-(2 / N)$, (4) holds with $\sigma \in(0,1)$, $\Gamma_{1}, \Gamma_{2}>0$ and $\alpha_{1}<1-((1-m) N / 4), \alpha_{2}<1-((1-m) N /$ 4). Then, there exist $p>1$ and $q>1$, such that

$$
\int_{0}^{t}\|u\|_{L^{p}(\Omega)}^{q} \leq C(t+1), \quad \text { for all } t \in\left[0, T_{\max }\right) .
$$

By (26), we have

Proof. By the Gagliardo-Nirenberg inequality, there exist $p>1$ and $q>1$, such that 


$$
\begin{aligned}
\|u\|_{L^{p}(\Omega)}^{q}= & \left\|u\left(\left(m-2 \alpha_{1}+1\right) / 2\right)\right\|_{L^{\left(2 p /\left(m-2 \alpha_{1}+1\right)\right)}(\Omega)}^{\left(2 q /\left(m-2 \alpha_{1}+1\right)\right)} \\
\leq & c_{8}\left\|\nabla u\left(\left(m-2 \alpha_{1}+1\right) / 2\right)\right\|_{L^{2}(\Omega)}^{\left(2 q /\left(m-2 \alpha_{1}+1\right)\right) \theta_{2}}\left\|u^{\left(\left(m-2 \alpha_{1}+1\right) / 2\right)}\right\|_{L^{\left(4\left(1-\alpha_{1}\right) /\left(m-2 \alpha_{1}+1\right)\right)(\Omega)}}^{\left(2 q /\left(m-2 \alpha_{1}+1\right)\right)\left(1-\theta_{2}\right)} \\
& +c_{8}\left\|u^{\left(\left(m-2 \alpha_{1}+1\right) / 2\right)}\right\|_{L^{\left(4\left(1-\alpha_{1}\right) /\left(m-2 \alpha_{1}+1\right)\right)}(\Omega)}^{\left(2 q /\left(m-2 \alpha_{1}+1\right)\right)}, \\
\|u\|_{L^{p}(\Omega)}^{q}= & \left\|u\left(\left(m-2 \alpha_{2}+1\right) / 2\right)\right\|_{L^{\left(2 p /\left(m-2 \alpha_{2}+1\right)\right)}(\Omega)}^{\left(2 q /\left(m-2 \alpha_{2}+1\right)\right)} \\
\leq & c_{9}\left\|\nabla u\left(\left(m-2 \alpha_{2}+1\right) / 2\right)\right\|_{L^{2}(\Omega)}^{\left(2 q /\left(m-2 \alpha_{2}+1\right)\right) \theta_{3}}\left\|u^{\left(\left(m-2 \alpha_{2}+1\right) / 2\right)}\right\|_{L^{\left(4\left(1-\alpha_{2}\right) /\left(m-2 \alpha_{2}+1\right)\right)}(\Omega)}^{\left(2 q /\left(m-2 \alpha_{2}+1\right)\right)\left(1-\theta_{3}\right)} \\
& +c_{9}\left\|u^{\left(\left(m-2 \alpha_{2}+1\right) / 2\right)}\right\|_{L^{\left(4\left(1-\alpha_{2}\right) /\left(m-2 \alpha_{2}+1\right)\right)}(\Omega)}^{\left(2 q /\left(m-2 \alpha_{2}+1\right)\right)},
\end{aligned}
$$

for all $t \in\left(0, T_{\max }\right)$ with some $c_{5}, c_{6}>0$ and

$$
\theta_{2}=\frac{\left(\left(m-2 \alpha_{1}+1\right) N / 4\left(1-\alpha_{1}\right)\right)-\left(\left(m-2 \alpha_{1}+1\right) N / 2 p\right)}{1-(N / 2)+\left(\left(m-2 \alpha_{1}+1\right) N / 4\left(1-\alpha_{1}\right)\right)} \in(0,1) \text {, }
$$

$$
\int_{\Omega} u^{2-2 \alpha_{1}} \leq c_{10}, \quad \text { for all } t \in\left(0, T_{\max }\right) \text {, }
$$

as well as

$$
\begin{aligned}
& \int_{0}^{t} \int_{\Omega} u^{m-2 \alpha_{1}-1}|\nabla u|^{2} \leq c_{11}(1+t), \\
& \int_{0}^{t} \int_{\Omega} u^{m-2 \alpha_{2}-1}|\nabla u|^{2} \leq c_{11}(1+t),
\end{aligned}
$$

$\theta_{3}=\frac{\left(\left(m-2 \alpha_{2}+1\right) N / 4\left(1-\alpha_{2}\right)\right)-\left(\left(m-2 \alpha_{2}+1\right) N / 2 p\right)}{1-(N / 2)+\left(\left(m-2 \alpha_{2}+1\right) N / 4\left(1-\alpha_{2}\right)\right)} \in(0,1)$.

In accordance with Lemma 5, there exist some $c_{10}, c_{11}>0$, such that

Substituting above four inequalities into (34) and (35), we have

$$
\begin{aligned}
& \int_{0}^{t}\|u\|_{L^{p}(\Omega)}^{q} \leq c_{8} \int_{0}^{t}\left\|\nabla u^{\left(\left(m-2 \alpha_{1}+1\right) / 2\right)}\right\|_{L^{2}(\Omega)}^{\left(2 q /\left(m-2 \alpha_{1}+1\right)\right) \theta_{2}}\left\|u^{\left(\left(m-2 \alpha_{1}+1\right) / 2\right)}\right\|_{L^{\left(4\left(1-\alpha_{1}\right) /\left(m-2 \alpha_{1}+1\right)\right)}(\Omega)}^{\left(2 q /\left(m-2 \alpha_{1}+1\right)\right)\left(1-\theta_{2}\right)} \\
& +c_{8} \int_{0}^{t}\left\|\mathcal{u}^{\left(\left(m-2 \alpha_{1}+1\right) / 2\right)}\right\|_{L^{\left(4\left(1-\alpha_{1}\right) /\left(m-2 \alpha_{1}+1\right)\right)}(\Omega)}^{\left(2 q /\left(m-2 \alpha_{1}+1\right)\right)\left(1-\theta_{2}\right)} \\
& \leq c_{8} c_{10}^{\left(q\left(1-\theta_{2}\right) / 2\left(m-2 \alpha_{1}+1\right)\right)} \int_{0}^{t}\left\|\nabla u^{\left(\left(m-2 \alpha_{1}+1\right) / 2\right)}\right\|_{L^{2}(\Omega)}^{\left(2 q /\left(m-2 \alpha_{1}+1\right)\right) \theta_{2}}+c_{8} c_{10}^{\left(q / 2\left(m-2 \alpha_{1}+1\right)\right)} \cdot t \\
& \leq c_{12} t+c_{12} \text {, } \\
& \int_{0}^{t}\|u\|_{L^{p}(\Omega)}^{q} \leq c_{9} \int_{0}^{t}\left\|\nabla u^{\left(\left(m-2 \alpha_{1}+1\right) / 2\right)}\right\|_{L^{2}(\Omega)}^{\left(2 q /\left(m-2 \alpha_{2}+1\right)\right) \theta_{3}}\left\|u\left(\left(m-2 \alpha_{2}+1\right) / 2\right)\right\|_{L^{\left(4\left(1-\alpha_{2}\right) /\left(m-2 \alpha_{2}+1\right)\right)}(\Omega)}^{\left(2 q /\left(m-2 \alpha_{2}+1\right)\right)\left(1-\theta_{3}\right)} \\
& +c_{9} \int_{0}^{t}\left\|u^{\left(\left(m-2 \alpha_{2}+1\right) / 2\right)}\right\|_{L^{\left(4\left(1-\alpha_{2}\right) /\left(m-2 \alpha_{2}+1\right)\right)}(\Omega)}^{\left(2 q /\left(m-2 \alpha_{2}+1\right)\right)\left(1-\theta_{3}\right)} \\
& \leq c_{9} c_{11}^{\left(q\left(1-\theta_{3}\right) / 2\left(m-2 \alpha_{2}+1\right)\right)} \int_{0}^{t}\left\|\nabla u^{\left(\left(m-2 \alpha_{2}+1\right) / 2\right)}\right\|_{L^{2}(\Omega)}^{\left(2 q /\left(m-2 \alpha_{2}+1\right)\right) \theta_{2}}+c_{9} c_{11}^{\left(q / 2\left(m-2 \alpha_{2}+1\right)\right)} \cdot t \\
& \leq c_{13} t+c_{13} \text {, }
\end{aligned}
$$

for all $t \in\left(0, T_{\max }\right)$ with some $c_{12}, c_{13}>0$, where we used the fact that $\left(q \theta_{2} /\left(m-2 \alpha_{1}+1\right)\right)<2$ and $\left(q \theta_{3} /\left(m-2 \alpha_{2}+1\right)\right)<$ 2 , and the proof similar to Lemma 2.6 in [13]. The proof is complete. that

$$
\begin{aligned}
& n_{1}(x, t) \leq c_{14}(1+t), \\
& n_{2}(x, t) \leq c_{15}(1+t),
\end{aligned}
$$

with some $c_{14}, c_{15}>0$. We claim that for any $N \geq 2$ and for all $T \in\left(0, T_{\max }\right)$, there exist some $\Upsilon_{1}(T), \Upsilon_{2}(T)>0$, such that $n_{1}(x, t) \leq \Upsilon_{1}(T)$ and $n_{2}(x, t) \leq \Upsilon_{2}(T)$ in $(0, T) \times \Omega$. This 
together with the definition of $n_{1}, n_{2}$ entails $v \geq\left\|v_{0}\right\|$ $L^{\infty}(\Omega)^{-\Upsilon_{1}(T)}$ and $w \geq\left\|w_{0}\right\|_{L^{\infty}(\Omega)} e^{-\Upsilon_{2}(T)}$ in $(0, T) \times \Omega$. With this in hand, we next consider system (3).

Lemma 7. Let $N \geq 2, T \in\left(0, T_{\max }\right)$ and suppose that $\min \{1-(2 / N), 2-(6 /(N+2))\}<m \leq 2-(2 / N)$, (4) holds with $\sigma \in(0,1), \Gamma_{1}, \Gamma_{2}>0$, and

$$
\begin{aligned}
& \alpha_{1}<1-\frac{(1-m) N}{4}, \\
& \alpha_{2}<1-\frac{(1-m) N}{4} .
\end{aligned}
$$

Then, for all $p>1$, there exists $C:=C(T)>0$, such that

$$
\frac{\mathrm{d}}{\mathrm{d} t} \int_{\Omega} u^{p}+\frac{2 p(p-1)}{(p+m-1)^{2}} \int_{\Omega}\left|\nabla u^{((p+m-1) / 2)}\right|^{2} \leq C \int_{\Omega} u^{p+2 \alpha_{1}-m-1}|\nabla v|^{2}+C \int_{\Omega} u^{p+2 \alpha_{2}-m-1}|\nabla w|^{2} .
$$

Proof. Multiplying the first equation in (3) by $p u^{p-1}$, integrating by parts and Young's inequality, we have

$$
\begin{aligned}
\frac{\mathrm{d}}{\mathrm{d} t} \int_{\Omega} u^{p}= & p \int_{\Omega} u^{p-1}\left(\nabla \cdot\left((u+1)^{m-1} \nabla u\right)+\nabla \cdot\left(\frac{\phi_{1}(u)}{v} \nabla v\right)-\nabla \cdot\left(\frac{\phi_{2}(u)}{w} \nabla w\right)\right) \\
\leq & -p(p-1) \int_{\Omega} u^{p+m-3}|\nabla u|^{2}+p(p-1) \int_{\Omega} u^{p-2} \frac{\phi_{1}(u)}{v} \nabla u \cdot \nabla v \\
& -p(p-1) \int_{\Omega} u^{p-2} \frac{\phi_{2}(u)}{w} \nabla u \cdot \nabla w \\
\leq & -p(p-1) \int_{\Omega} u^{p+m-3}|\nabla u|^{2}+\frac{p(p-1)}{4} \int_{\Omega} u^{p-2}|\nabla u|^{2} \\
& +p(p-1) \int_{\Omega} u^{p-m-1} \frac{\phi_{1}^{2}(u)}{v^{2}}|\nabla v|^{2}+\frac{p(p-1)}{4} \int_{\Omega} u^{p+m-3}|\nabla u|^{2} \\
& +p(p-1) \int_{\Omega} u^{p-m-1} \frac{\phi_{2}^{2}(u)}{w^{2}}|\nabla w|^{2} \\
\leq & -\frac{p(p-1)}{2} \int_{\Omega} u^{p+m-3}|\nabla u|^{2}+\frac{p(p-1) \Gamma_{1}^{2} e^{2 Y_{1}(T)}}{\left\|v_{0}\right\|_{L^{\infty}(\Omega)}^{2}} \int_{\Omega} u^{p+2 \alpha_{1}-2}|\nabla v|^{2} \\
& +\frac{p(p-1) \Gamma_{2}^{2} e^{2 \Upsilon_{2}(T)}}{\left\|w_{0}\right\|_{L^{\infty}(\Omega)}^{2}} \int_{\Omega} u^{p+2 \alpha_{2}-2}|\nabla w|^{2},
\end{aligned}
$$

for all $t \in(0, T)$, here we used the fact that $v \geq\left\|v_{0}\right\|_{L^{\infty}(\Omega)} e^{-\Upsilon_{1}(T)}$ and $w \geq\left\|w_{0}\right\|_{L^{\infty}(\Omega)} e^{-\Upsilon_{2}(T)}$ in $(0, T) \times \Omega$. We obtain (42) if we let

$$
C:=\max \left\{\frac{p(p-1) \Gamma_{1}^{2} e^{2 \Upsilon_{1}(T)}}{\left\|v_{0}\right\|_{L^{\infty}(\Omega)}^{2}}, \frac{p(p-1) \Gamma_{2}^{2} e^{2 \Upsilon_{2}(T)}}{\left\|w_{0}\right\|_{L^{\infty}(\Omega)}^{2}}\right\} .
$$

The proof is complete.
Applying the same arguments as in the proof of Lemma 2.9 and Lemma 2.10 in [13], we have the following Lemma.

Lemma 8. Assume that $\Omega \subset \mathbb{R}^{N}(N \geq 1)$ be a bounded domain with smooth boundary. Then, for all $q \geq 2$, there exists $C>0$, such that

$$
\begin{gathered}
\frac{1}{q} \frac{\mathrm{d}}{\mathrm{d} t} \int_{\Omega}|\nabla v|^{2 q}+\int_{\Omega}|\nabla v|^{2 q-2}\left|D^{2} v\right|^{2} \leq C \int_{\Omega} u^{2}|\nabla v|^{2 q-2}+C, \quad \text { for all } t \in\left(0, T_{\max }\right), \\
\frac{1}{q} \frac{\mathrm{d}}{\mathrm{d} t} \int_{\Omega}|\nabla w|^{2 q}+\int_{\Omega}|\nabla w|^{2 q-2}\left|D^{2} w\right|^{2} \leq C \int_{\Omega} u^{2}|\nabla w|^{2 q-2}+C, \quad \text { for all } t \in\left(0, T_{\max }\right) .
\end{gathered}
$$


Lemma 9. Let $N \geq 2$. Assume $\min \{1-(2 / N), 2-$ $(6 /(N+2))\}<m \leq 2-(2 / N)$, (3) holds with $\sigma \in(0,1)$, $\Gamma_{1}, \Gamma_{2}>0$, and

$$
\begin{aligned}
& \alpha_{1}<1-\frac{(1-m) N}{4}, \\
& \alpha_{2}<1-\frac{(1-m) N}{4} .
\end{aligned}
$$

Let $p$ and $q$ be the same as in Lemmas 7 and 8 . Then, for all $T \in\left(0, T_{\max }\right)$, one can find some $C>0$ fulfilling

$$
\begin{array}{r}
\|u(x, t)\|_{L^{p}(\Omega)} \leq C, \\
\|\nabla v(x, t)\|_{L^{2 q}(\Omega)} \leq C,
\end{array}
$$

as well as

$$
\|\nabla w(x, t)\|_{L^{2 q}(\Omega)} \leq C .
$$

Proof. We first consider the dimensional $N \geq 2$. Combining with Lemmas 7 and 8 , there exists some $c_{16}:=c_{16}(T)>0$, such that

$$
\begin{aligned}
& \frac{\mathrm{d}}{\mathrm{d} t}\left(\int_{\Omega} u^{p}+\frac{1}{q} \int_{\Omega}|\nabla v|^{2 q}+\frac{1}{q} \int_{\Omega}|\nabla w|^{2 q}\right)+\frac{2 p(p-1)}{(p+m-1)^{2}} \int_{\Omega}\left|\nabla u^{((p+m-1) / 2)}\right|^{2} \\
& \quad+\int_{\Omega}|\nabla v|^{2 q-2}\left|D^{2} v\right|^{2}+\int_{\Omega}|\nabla w|^{2 q-2}\left|D^{2} w\right|^{2} \\
& \leq c_{16} \int_{\Omega} u^{p+2 \alpha_{1}-m-1}|\nabla v|^{2}+c_{16} \int_{\Omega} u^{p+2 \alpha_{2}-m-1}|\nabla w|^{2} \\
& \quad+c_{16} \int_{\Omega} u^{2}|\nabla v|^{2 q-2}+c_{16} \int_{\Omega} u^{2}|\nabla w|^{2 q-2}
\end{aligned}
$$

for all $t \in(0, T)$. By the Hölder inequality, we have

$$
\begin{gathered}
\int_{\Omega} u^{p+2 \alpha_{1}-m-1}|\nabla v|^{2} \leq\left(\int_{\Omega} u^{\left(p+2 \alpha_{1}-m-1\right) \tau_{1}}\right)^{\left(1 / \tau_{1}\right)}\left(\int_{\Omega}|\nabla v|^{2 \tau_{1}^{\prime}}\right)^{\left(1 / \tau_{1}^{\prime}\right)}, \\
\int_{\Omega} u^{p+2 \alpha_{2}-m-1}|\nabla w|^{2} \leq\left(\int_{\Omega} u^{\left(p+2 \alpha_{2}-m-1\right) \tau_{2}}\right)^{\left(1 / \tau_{2}\right)}\left(\int_{\Omega}|\nabla w|^{2 \tau_{2}^{\prime}}\right)^{\left(1 / \tau_{2}^{\prime}\right)},
\end{gathered}
$$

for all $t \in(0, T)$, where $\tau_{1}, \tau_{2}:=((q+1) / q)$ and $\tau_{1}^{\prime}, \tau_{2}^{\prime}:=q+1$. Moreover, by the Gagliardo-Nirenberg inequality, there exist some constants $c_{17}, c_{18}>0$, such that

$$
\begin{aligned}
\left(\int_{\Omega} u^{\left(p+2 \alpha_{1}-m-1\right) \tau_{1}}\right)^{\left(1 / \tau_{1}\right)}= & \left\|u^{((p+m-1) / 2)}\right\|_{L^{\left(2\left(p+2 \alpha_{1}-m-1\right) \tau_{1} /(p+m-1)\right)}(\Omega)}^{\left(2\left(p+2 \alpha_{1}-m-1\right) /(p+m-1)\right)} \\
\leq & c_{17}\left\|\nabla u^{((p+m-1) / 2)}\right\|_{L^{2}(\Omega)}^{\left(2\left(p+2 \alpha_{1}-m-1\right) / p\right) \theta_{4}}\left\|u^{((p+m-1) / 2)}\right\|_{L^{\left(2\left(2-2 \alpha_{1}\right) /(p+m-1)\right)}(\Omega)}^{\left(2\left(p+2 \alpha_{1}-m-1\right) /(p+m-1)\right)\left(1-\theta_{4}\right)} \\
& +c_{17}\left\|u^{((p+m-1) / 2)}\right\|_{L^{\left(2\left(2-2 \alpha_{1}\right) /(p+m-1)\right)}(\Omega)}^{\left(2\left(p+2 \alpha_{1}-m-1\right) /(p+m-1)\right)}, \\
\left(\int_{\Omega} u^{\left(p+2 \alpha_{2}-m-1\right) \tau_{1}}\right)^{\left(1 / \tau_{2}\right)}= & \left\|u^{((p+m-1) / 2)}\right\|_{L^{\left(2\left(p+2 \alpha_{2}-m-1\right) \tau_{2} /(p+m-1)\right)}(\Omega)}^{\left(2\left(p+2 \alpha_{2}-m-1\right) /(p+m-1)\right)} \\
\leq & c_{18}\left\|\nabla u^{((p+m-1) / 2)}\right\|_{L^{2}(\Omega)}^{\left(2\left(p+2 \alpha_{2}-m-1\right) / p\right) \theta_{5}}\left\|u^{((p+m-1) / 2)}\right\|_{L^{\left(2\left(2-2 \alpha_{2}\right) /(p+m-1)\right)}(\Omega)}^{\left(2\left(p+2 \alpha_{2}-m-1\right) /(p+m-1)\right)\left(1-\theta_{5}\right)} \\
& +c_{18}\left\|u^{((p+m-1) / 2)}\right\|_{L^{\left(2\left(2-2 \alpha_{2}\right) /(p+m-1)\right)}(\Omega)}^{\left(2\left(p+2 \alpha_{2}-m-1\right) /(p+m-1)\right)},
\end{aligned}
$$


with

$$
\begin{aligned}
& \theta_{4}=\frac{\left((p+m-1) N / 2\left(2-2 \alpha_{1}\right)\right)-\left((p+m-1) N / 2\left(p+2 \alpha_{1}-m-1\right) \tau_{1}\right)}{1-(N / 2)+\left((p+m-1) N / 2\left(2-2 \alpha_{1}\right)\right)} \in(0,1), \\
& \theta_{5}=\frac{\left((p+m-1) N / 2\left(2-2 \alpha_{2}\right)\right)-\left((p+m-1) N / 2\left(p+2 \alpha_{2}-m-1\right) \tau_{2}\right)}{1-(N / 2)+\left((p+m-1) N / 2\left(2-2 \alpha_{2}\right)\right)} \in(0,1)
\end{aligned}
$$

the reason analogous to (2.42) and (2.43) in ([13], Lemma

2.11). Therefore, combining with (20) and (52), we get

$$
\begin{aligned}
& \left(\int_{\Omega} u^{\left(p+2 \alpha_{1}-m-1\right) \tau_{1}}\right)^{\left(1 / \tau_{1}\right)} \leq c_{19}\left(\int_{\Omega}\left|\nabla u^{((p+m-1) / 2)}\right|^{2}\right)^{\left(\left(\left(N\left(p+2 \alpha_{1}-m-1\right) / 4\left(1-\alpha_{1}\right)\right)-\left(N / 2 \tau_{1}\right)\right) /\left(1-(N / 2)+\left((p+m-1) N / 4\left(1-\alpha_{1}\right)\right)\right)\right)}+c_{19}, \\
& \left(\int_{\Omega} u^{\left(p+2 \alpha_{2}-m-1\right) \tau_{2}}\right)^{\left(1 / \tau_{2}\right)} \leq c_{20}\left(\int_{\Omega}\left|\nabla u^{((p+m-1) / 2)}\right|^{2}\right)^{\left(\left(\left(N\left(p+2 \alpha_{2}-m-1\right) / 4\left(1-\alpha_{2}\right)\right)-\left(N / 2 \tau_{2}\right)\right) /\left(1-(N / 2)+\left((p+m-1) N / 4\left(1-\alpha_{2}\right)\right)\right)\right)}+c_{20},
\end{aligned}
$$

for all $t \in(0, T)$, where $c_{19}:=c_{17} C_{3}^{\left(\left(p+2 \alpha_{1}-m-1\right) / 2\left(1-\alpha_{1}\right)\right)}$ and $c_{20}:=c_{18} C_{4}^{\left(\left(p+2 \alpha_{2}-m-1\right) / 2\left(1-\alpha_{2}\right)\right)}$. Along with (55), (56) follows from ([13], Lemma 2.9), (50), and (51), and we deduce

$$
\begin{gathered}
\int_{\Omega} u^{p+2 \alpha_{1}-m-1}|\nabla v|^{2} \leq c_{21}\left(\int_{\Omega}\left|\nabla u^{((p+m-1) / 2)}\right|^{2}\right)^{\left(\left(\left(N\left(p+2 \alpha_{1}-m-1\right) / 4\left(1-\alpha_{1}\right)\right)-\left(N / 2 \tau_{1}\right)\right) /\left(1-(N / 2)+\left((p+m-1) N / 4\left(1-\alpha_{1}\right)\right)\right)\right)}\left(\int_{\Omega}|\nabla v|^{2 q-2}\left|D^{2} v\right|^{2}\right)^{(1 /(q+1))} \\
+c_{21}\left(\int_{\Omega}|\nabla v|^{2 q-2}\left|D^{2} v\right|^{2}\right)^{(1 /(q+1))} \\
\int_{\Omega} u^{p+2 \alpha_{2}-m-1}|\nabla w|^{2} \leq \\
c_{22}\left(\int_{\Omega}\left|\nabla u^{((p+m-1) / 2)}\right|^{2}\right)^{\left(\left(\left(N\left(p+2 \alpha_{2}-m-1\right) / 4\left(1-\alpha_{2}\right)\right)-\left(N / 2 \tau_{2}\right)\right) /\left(1-(N / 2)+\left((p+m-1) N / 4\left(1-\alpha_{2}\right)\right)\right)\right)} \\
\cdot\left(\int_{\Omega}|\nabla w|^{2 q-2}\left|D^{2} w\right|^{2}\right)^{(1 /(q+1))}+c_{22}\left(\int_{\Omega}|\nabla w|^{2 q-2}\left|D^{2} w\right|^{2}\right)^{(1 /(q+1))}
\end{gathered}
$$


for all $t \in(0, T)$, with some constants $c_{21}, c_{22}>0$ determined by $T$. Similar to (2.45) in ([13], Lemma 2.11), we know that

$$
\begin{aligned}
& \frac{\left(N\left(p+2 \alpha_{1}-m-1\right) / 4\left(1-\alpha_{1}\right)\right)-(q N / 2(q+1))}{1-(N / 2)+\left((p+m-1) N / 4\left(1-\alpha_{1}\right)\right)}+\frac{1}{q+1}<1, \\
& \frac{\left(N\left(p+2 \alpha_{2}-m-1\right) / 4\left(1-\alpha_{2}\right)\right)-(q N / 2(q+1))}{1-(N / 2)+\left((p+m-1) N / 4\left(1-\alpha_{2}\right)\right)}+\frac{1}{q+1}<1 .
\end{aligned}
$$

Therefore, using the Young inequality, we derive

$$
\begin{aligned}
& c_{21}\left(\int_{\Omega}\left|\nabla u^{((p+m-1) / 2)}\right|^{2}\right)^{\left(\left(\left(N\left(p+2 \alpha_{1}-m-1\right) / 4\left(1-\alpha_{1}\right)\right)-\left(N / 2 \tau_{1}\right)\right) /\left(1-(N / 2)+\left((p+m-1) N / 4\left(1-\alpha_{1}\right)\right)\right)\right)}\left(\int_{\Omega}|\nabla v|^{2 q-2}\left|D^{2} v\right|^{2}\right)^{(1 /(q+1))} \\
& \leq \frac{p-1}{4 c_{16} p} \int_{\Omega}\left|\nabla u^{((p+m-1) / 2)}\right|^{2}+\frac{1}{8 c_{16}} \int_{\Omega}|\nabla v|^{2 q-2}\left|D^{2} v\right|^{2}+c_{23}, \\
& c_{22}\left(\int_{\Omega}\left|\nabla u^{((p+m-1) / 2)}\right|^{2}\right)^{\left(\left(\left(N\left(p+2 \alpha_{2}-m-1\right) / 4\left(1-\alpha_{2}\right)\right)-\left(N / 2 \tau_{2}\right)\right) /\left(1-(N / 2)+\left((p+m-1) N / 4\left(1-\alpha_{2}\right)\right)\right)\right)}\left(\int_{\Omega}|\nabla w|^{2 q-2}\left|D^{2} w\right|^{2}\right)^{\frac{1}{q+1}} \\
& \leq \frac{p-1}{4 c_{16} p} \int_{\Omega}\left|\nabla u^{((p+m-1) / 2)}\right|^{2}+\frac{1}{8 c_{16}} \int_{\Omega}|\nabla w|^{2 q-2}\left|D^{2} w\right|^{2}+c_{24},
\end{aligned}
$$

for all $t \in(0, T)$, with some constants $c_{23}, c_{24}>0$ determined by $T$. Once more, employing Young's inequality, there exist some constants $c_{25}, c_{26}>0$ determined by $T$, such that

$$
c_{21}\left(\int_{\Omega}|\nabla v|^{2 q-2}\left|D^{2} v\right|^{2}\right)^{(1 /(q+1))} \leq \frac{1}{8 c_{16}} \int_{\Omega}|\nabla v|^{2 q-2}\left|D^{2} v\right|^{2}+c_{25},
$$

$$
\begin{aligned}
\int_{\Omega} u^{p+2 \alpha_{1}-2}|\nabla v|^{2} \leq & \frac{p-1}{4 c_{16} p} \int_{\Omega}\left|\nabla u^{((p+m-1) / 2)}\right|^{2} \\
& +\frac{1}{4 c_{16}} \int_{\Omega}|\nabla v|^{2 q-2}\left|D^{2} v\right|^{2}+c_{23}+c_{25} \\
\int_{\Omega} u^{p+2 \alpha_{2}-2}|\nabla w|^{2} \leq & \frac{p-1}{4 c_{16} p} \int_{\Omega}\left|\nabla u^{((p+m-1) / 2)}\right|^{2} \\
& +\frac{1}{4 c_{16}} \int_{\Omega}|\nabla w|^{2 q-2}\left|D^{2} w\right|^{2}+c_{24}+c_{26}
\end{aligned}
$$

as well as

for all $t \in(0, T)$. Combining with (57)-(63), we obtain

for all $t \in(0, T)$. Analogous to (64) and (65), we have

$$
\begin{aligned}
& \int_{\Omega} u^{2}|\nabla v|^{2 q-2} \leq \frac{p-1}{4 c_{16} p} \int_{\Omega}\left|\nabla u^{((p+m-1) / 2)}\right|^{2}+\frac{1}{4 c_{16}} \int_{\Omega}|\nabla v|^{2 q-2}\left|D^{2} v\right|^{2}+c_{27} \\
& \int_{\Omega} u^{2}|\nabla w|^{2 q-2} \leq \frac{p-1}{4 c_{16} p} \int_{\Omega}\left|\nabla u^{((p+m-1) / 2)}\right|^{2}+\frac{1}{4 c_{16}} \int_{\Omega}|\nabla w|^{2 q-2}\left|D^{2} w\right|^{2}+c_{28}
\end{aligned}
$$

for all $t \in(0, T)$, with some constants $c_{27}, c_{28}>0$ determined by $T$. By the Gagliardo-Nirenberg inequality, there exists a constant $c_{29}>0$, such that

$$
\int_{\Omega} u^{p} \leq \frac{p-1}{2 c_{16} p} \int_{\Omega}\left|\nabla u^{((p+m-1) / 2)}\right|^{2}+c_{29}
$$

Together with (49) and (64)-(68), we have

$$
\frac{\mathrm{d}}{\mathrm{d} t}\left(\int_{\Omega} u^{p}+\frac{1}{q} \int_{\Omega}|\nabla v|^{2 q}+\frac{1}{q} \int_{\Omega}|\nabla w|^{2 q}\right) \leq c_{30}
$$

for all $t \in(0, T)$ with some constants $c_{30}>0$. The proof is complete. 
Finally, we prove the main theorem.

Proof. of Theorem 1. From Lemma 9 and the Moser-Alikakos iterative technique in Lemma A.1 of [30], we have for all $T \in\left(0, T_{\max }\right)$,

$$
\|u(., t)\|_{L^{\infty}(\Omega)} \leq C,
$$

for all $t \in(0, T)$ with some constant $C>0$ determined by $T$. Thus, the global existence is a consequence of (70) and the extensibility criterion provided by Lemma 1.

\section{Data Availability}

No data were used to support this study.

\section{Conflicts of Interest}

The author declares that there are no conflicts of interest.

\section{Acknowledgments}

The study was supported by the NSF of China (11801154).

\section{References}

[1] E. F. Keller and L. A. Segel, "Initiation of slime mold aggregation viewed as an instability," Journal of Theoretical Biology, vol. 26, no. 3, pp. 399-415, 1970.

[2] M. A. Herrero and J. J. L. Velázquez, "A blow-up mechanism for a chemotaxis model," Ann Scuola Normale Superiore Pisa, vol. 24, pp. 633-683, 1997.

[3] T. Nagai, T. Senba, and K. Yoshida, "Application of the Trudinger-Moser inequality to a parabolic system of chemotaxis," Funkcial. Ekvac.vol. 40, pp. 411-433, 1997.

[4] K. Osaki and A. Yagi, "Finite dimensional attractor for onedimensional Keller-Segel equations," Funkcialaj Ekvacioj, vol. 44, pp. 441-469, 2001.

[5] M. Winkler, "Aggregation vs. global diffusive behavior in the higher-dimensional Keller-Segel model," Journal of Differential Equations, vol. 248, no. 12, pp. 2889-2905, 2010.

[6] M. Winkler, "Finite-time blow-up in the higher-dimensional parabolic-parabolic Keller-Segel system," Journal de Mathématiques Pures et Appliquées, vol. 100, no. 5, pp. 748767, 2013.

[7] E. F. Keller and L. A. Segel, "Traveling bands of chemotactic bacteria: a theoretical analysis," Journal of Theoretical Biology, vol. 30, no. 2, pp. 235-248, 1971.

[8] M. Winkler, "The two-dimensional Keller-Segel system with singular sensitivity and signal absorption Global large-data solutions and their relaxation properties," Mathematical Models and Methods in Applied Sciences, vol. 26, no. 5, pp. 987-1024, 2016.

[9] J. Lankeit and G. Viglialoro, "Global existence and boundedness of solutions to a chemotaxis-consumption model with singular sensitivity," Acta Applicandae Mathematicae, vol. 167, no. 1, pp. 75-97, 2020.

[10] E. Lankeit and J. Lankeit, "On the global generalized solvability of a chemotaxis model with signal absorption and logistic growth terms," Nonlinearity, vol. 32, no. 5, pp. 1569-1596, 2019.

[11] X. Zhao and S. Zheng, "Global existence and asymptotic behavior to a chemotaxis-consumption system with singular sensitivity and logistic source," Nonlinear Analysis: Real World Applications, vol. 42, pp. 120-139, 2018.

[12] X. Zhao and S. Zheng, "Asymptotic behavior to a chemotaxisconsumption system with singular sensitivity," Mathematical Methods in the Applied Sciences, vol. 41, no. 4, pp. 1-10, 2018.

[13] D. Liu, "Global classical solution to a chemotaxis consumption model with singular sensitivity," Nonlinear Analysis: Real World Applications, vol. 41, pp. 497-508, 2018.

[14] J. Lankeit, "Locally bounded global solutions to a chemotaxis consumption model with singular sensitivity and nonlinear diffusion," Journal of Differential Equations, vol. 262, no. 7, pp. 4052-4084, 2017.

[15] M. Luca, A. Chavez-Ross, L. Edelstein-Keshet, and A. Mogilner, "Chemotactic signaling, microglia, and alzheimer's disease senile plaques: is there a connection?" Bulletin of Mathematical Biology, vol. 65, no. 4, pp. 693-730, 2003.

[16] H. Jiu and Z. Wang, "Boundedness, blowup and critical mass phenomenon in competing chemotaxis," Journal of Differential Equations, vol. 260, pp. 162-196, 2016.

[17] J. Xu, Z. Liu, and S. Shi, "Large time behavior of solutions for the attraction-repulsion Keller-Segel system with large initial data," Applied Mathematics Letters, vol. 87, pp. 13-19, 2019.

[18] Y. Li and Y. Li, "Blow-up of nonradial solutions to attractionrepulsion chemotaxis system in two dimensions," Nonlinear Analysis: Real World Applications, vol. 30, pp. 170-183, 2016.

[19] H. Yu, Q. Guo, and S. Zheng, "Finite time blow-up of nonradial solutions in an attraction-repulsion chemotaxis system," Nonlinear Analysis: Real World Applications, vol. 34, pp. 335-342, 2017.

[20] H.-Y. Jin, "Boundedness of the attraction-repulsion KellerSegel system," Journal of Mathematical Analysis and Applications, vol. 422, no. 2, pp. 1463-1478, 2015.

[21] K. Lin, C. Mu, and Y. Gao, "Boundedness and blow up in the higher-dimensional attraction-repulsion chemotaxis system with nonlinear diffusion," Journal of Differential Equations, vol. 261, no. 8, pp. 4524-4572, 2016.

[22] J. Li and Y. Wang, "Repulsion effects on boundedness in the higher dimensional fully parabolic attraction-repulsion chemotaxis system," Journal of Mathematical Analysis and Applications, vol. 467, no. 2, pp. 1066-1079, 2018.

[23] G. Ren and B. Liu, "Global existence of bounded solutions for a quasilinear chemotaxis system with logistic source," Nonlinear Analysis: Real World Applications, vol. 46, pp. 545-582, 2019.

[24] G. Ren and B. Liu, "Global boundedness and asymptotic behavior in a quasilinear attraction-repulsion chemotaxis model with nonlinear signal production and logistic-type source," Mathematical Models and Methods in Applied Sciences, vol. 30, no. 13, pp. 2619-2689, 2020.

[25] G. Ren and B. Liu, "Global dynamics for an attraction-repulsion chemotaxis model with logistic source," Journal of Differential Equations, vol. 268, no. 8, pp. 4320-4373, 2020.

[26] Y. Dong and Z. Xiang, "Global large-data generalized solutions in a chemotactic movement with rotational flux caused by two stimuli," Nonlinear Analysis: Real World Applications, vol. 41, pp. 549-569, 2018.

[27] J. Yan and Y. Li, "Global generalized solutions to a KellerSegel system with nonlinear diffusion and singular sensitivity," Nonlinear Analysis, vol. 176, pp. 288-302, 2018.

[28] D. Gilbarg and N. S. Trudinger, Elliptic Partial Differential Equations of Second Order, Springer-Verlag, Berlin, Germany, 2001. 
[29] Y. Wang, "Global weak solutions in a three-dimensional Keller-Segel-Navier-Stokes system with subcritical sensitivity," Mathematical Models and Methods in Applied Sciences, vol. 27, no. 14, pp. 2745-2780, 2017.

[30] Y. Tao and M. Winkler, "Boundedness in a quasilinear parabolic-parabolic Keller-Segel system with subcritical sensitivity," Journal of Differential Equations, vol. 252, no. 1, pp. 692-715, 2012. 